\title{
On the Difficulty of the Transport of Electrically Charged Submicron Dust from the Earth's Surface to the High Ionosphere
}

\author{
N. Chandra Wickramasinghe ${ }^{1,2,3^{*}}$ and Michael J. Rycroft ${ }^{4.5^{* *}}$ \\ ${ }^{1}$ Buckingham Centre for Astrobiology, University of Buckingham, Buckingham MK18 1EG, UK, \\ ${ }^{2}$ Centre for Astrobiology, University of Ruhuna, Matara, Sri Lanka, \\ ${ }^{3}$ Institute for the Study of Panspermia and Astrobiology, Gifu, Japan, \\ ${ }^{4}$ Department of Electronic and Electrical Engineering, University of Bath, Bath BA2 7AY, UK, \\ ${ }^{5}$ CAESAR Consultancy, 35 Millington Road, Cambridge CB3 9HW, UK. \\ *Email: ncwick@gmail.com \\ **Email: michaelrycroft@btinternet.com
}

\begin{abstract}
The transport of electrically charged bacterial-sized dust particles from the stratosphere to the high ionosphere is discussed. With the maximum electric field associated with the Global Electric Circuit during both active thunderstorm and fair weather conditions, we show that electric forces are inadequate to overcome gravity in lofting dust particles from the stratosphere to heights of $\sim 400 \mathrm{~km}$ in the ionosphere, where they have recently been discovered at the orbit of the International Space Station.
\end{abstract}

Keywords: Dust, bacteria, atmosphere, stratosphere, ionosphere, electric field, panspermia.

\section{Introduction}

Dust in the form of particles in the radius range $10^{-6}-10^{-4} \mathrm{~cm}$ is widespread throughout the atmosphere and troposphere. The origin of this dust is predominantly from the Earth's surface including the oceans, and a peak aerosol density has been reported to be in the height range $2-4 \mathrm{~km}$. It is now well recognised that the terrestrial dust load includes a significant biological component in the form of bacteria and viruses, and moreover that this material is being continually cycled through a "microbiome" in the tropopause (Lynch et al, 2005; Reche et al, 2018) [1,2]. The average population density of such dust falls exponentially above the tropopause at $\sim 15 \mathrm{~km}$, where it reaches a local maximum, and by an altitude of $\sim 30 \mathrm{~km}$ it is essentially zero. The flux of solar ultra-violet radiation prevailing in the stratosphere at heights $30-50 \mathrm{~km}$ appears to be distinctly inhospitable for the long-term survival of bacteria (Khodadad et al, 2017; Smith, 2013) [3,4].

Bacteria have been isolated from the stratosphere over several years and the presumption has been that they are of extra-terrestrial origin (Imshenetsky et al, 1978; Wainwright et al, 2003; Grebennikova et al, 2018) $[5,6,7]$. The height distribution of stratospheric bacteria inferred from balloon sampling carried out in 2001 was interpreted as being consistent with in-falling particles rather than particles lofted from the ground (Narlikar et al, 2003) [8]. Higher up in the mesosphere, nanometre-sized grains are known to exist but these particles, which are responsible for nucleating ice particles in noctilucent clouds, are thought to result from the disruption of small, possibly fluffy, meteoroids.

Except during times of exceptionally energetic events such as powerful volcanic eruptions or (much more rarely) asteroid or comet impacts, dust from the surface cannot be naturally lofted into the stratosphere and above. The recent report of bacteria found at the orbit of the International Space Station (ISS) at $400 \mathrm{~km}$ altitude is a counter-example that needs to be understood [7]. In this note we discuss the possibility of electric fields contributing to the transport of charged bacteria and dust from lower levels in the atmosphere into the ionosphere; we show that they are too small to have any significant effect. 


\section{Electric Charge on a Dust Grain}

The well-recognised global electrical circuit (GEC) involves the motion of electrons and ions between the Earth's surface and the ionosphere driven mainly by thunderstorms (Rycroft et al, 2000) [9]. Upward directed electric fields exert upward forces on positively charged aerosol particles, including bacteria, and the magnitude of these forces needs to be determined. To this end we estimate the net charge on dust particles in the ionosphere.

A spherical dust particle of radius $\mathrm{r}$ immersed in the ionosphere will experience collisions with electrons and positive ions. The precise ionic composition varies with height and time, but for the purpose of our discussion we can assume $\mathrm{O}^{+}$to be a typical positive ion. The plasma temperature at a height of $\sim 200 \mathrm{~km}$ is in the range $\mathrm{T} \sim 1000-2000 \mathrm{~K}$, and the electron density is $10^{4}-10^{6} \mathrm{~cm}^{-3}$. If $\mathrm{v}_{\mathrm{e}}, \mathrm{m}_{\mathrm{e}}$ and $\mathrm{v}_{\mathrm{i}}, \mathrm{m}_{\mathrm{i}}$ are the mean speeds and masses of the electrons and ions respectively, the equipartition of energy between electrons and ions gives

$$
0.5 m_{e} v_{e}^{2} \cong 0.5 m_{i} v_{i}^{2}
$$

so that

$$
v_{e}=\sqrt{\left(m_{i} / m_{e}\right)} \times v_{i} \cong 172 v_{i}
$$

Electrons approaching a dust grain therefore have speeds $\sim 172$ times larger than ions, so it is reasonable to expect that the dust particle will acquire a net negative charge $\mathrm{Q}$ in order to maintain a balance between the rate of incidence of electrons and positive ions.

The procedure for calculating the equilibrium charge on a dust grain in the ionosphere is identical to that used for calculating the net electric charge on an interstellar grain in an HII region, equating incidence rates of positive ions and electrons and integrating over a Maxwell-Boltzmann distribution of velocities (Spitzer, 1941; Wickramasinghe, 1967) [10,11]. We shall now apply the numerical result from this analysis to calculate the charge of a dust particle in the ionosphere.

For a spherical dust particle of radius $\sim 3 \times 10^{-5} \mathrm{~cm}$ immersed in a plasma of temperature $10^{3} \mathrm{~K}$ (appropriate to the mid ionosphere), it can be shown that the negative equilibrium charge $\mathrm{Q}$ is given by

$$
Q \cong 10 e \cong 1.60 \times 10^{-18} \text { Coulomb }
$$

(Wickramasinghe, 1967) [11].

\section{Electric Field in the Stratosphere}

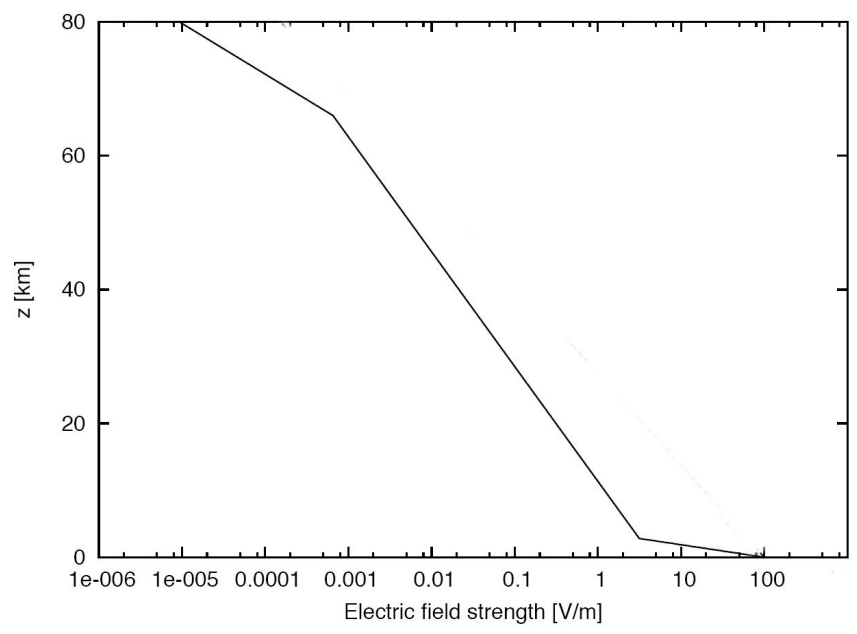

Figure 1. Best fitting model of Ampferer et al (2010)[12] of observations of fair weather electric field with altitude $\mathrm{z}$ through the troposphere, stratosphere, mesosphere and ionosphere.

In fair weather conditions remote from thunderstorms, the atmospheric electric field near the Earth's surface typically is about 150 Volts $\mathrm{m}^{-1}$ and is directed vertically in such a sense as to drive positive 
charges downward to the Earth. This field falls off exponentially with height to $<1 \mathrm{Volt} / \mathrm{m}$ at $30 \mathrm{~km}$. The electric field measured at the surface of the Earth varies widely in magnitude and direction and reverses its direction underneath active thunderstorms. Microorganisms and inorganic dust particles that have a net electric charge circulate in the lower atmosphere, and winds can transport them over large horizontal distances. Their vertical transport, however, would be controlled by updraughts/ downdraughts and the value of the vertical component of the electric field.

The fair weather electric field through the atmosphere has been extensively measured in recent years. The curve in Fig. 1 is a best fit to the experimental data modelled by Ampferer et al (2010) [12]. We note from Fig. 1 that already at $65 \mathrm{~km}$ the electric field $\mathrm{E}$ has fallen to below $10^{-3} \mathrm{~V} / \mathrm{m}$, and it continues to decline up to $80 \mathrm{~km}$ and beyond.

\section{Lofting Submicron-Sized Charged Dust}

The upward electric force exerted on such a positively charged particle in the atmospheric electric field of strength $\mathrm{E}$ is now

$$
F=E Q \cong 1.60 \times 10^{-18} E \text { Newton }
$$

using the result of the calculation leading to (3). With $\mathrm{E}$ being at the very most of the order $\mathrm{E} \approx 10^{-3}$ Volt/metre at $65 \mathrm{~km}$ (Ampferer et al, 2010; Rycroft et al, 2008) [12,9] we thus have a maximum upward electric force acting on the particle to be

$$
F \cong 1.6 \times 10^{-21} \text { Newton }
$$

This is to be compared with the downward gravitational force

$$
G \cong 4 \pi a^{3} \rho g / 3 \cong 2.3 \times 10^{-17} \text { Newton }
$$

for $\mathrm{a}=3 \times 10^{-5} \mathrm{~cm}, \rho=1 \mathrm{~g} \mathrm{~cm}^{-3}, \mathrm{~g}=981 \mathrm{~cm} \mathrm{~s}^{-2}$ (after converting to SI units).

Equations (5) and (6) give

$$
F / G \cong 7 \times 10^{-5}
$$

The conclusion is that mesospheric/ionospheric electric fields are inadequate by four powers of 10 at least to loft electrically charged micron-sized bacterial dust from the troposphere to the high ionosphere. Invoking "transient luminous events" - elves or sprites - does not offer much help either. Electric fields measured in such events are still small, of the order of $10^{-2}-10^{-3} \mathrm{~V} /$ metre (Thomas et al, 2008) [13]; so from equations (4-7) we still have a shortfall of several hundred in the $\mathrm{F} / \mathrm{G}$ ratio to permit gravitational escape. Moreover, such phenomena tend to be extremely fleeting, typically of a few milliseconds duration, and they are confined to just below the ionosphere.

\section{Thunderstorm Conditions}

Finally, we consider the possibility that charged dust particles (including charged bacteria) may be carried to the ionosphere ( $\sim 80 \mathrm{~km}$ altitude) by vertical updraughts above active thunderstorms which mainly occur at low latitudes, typically within 15 degrees of latitude $\mathrm{N}$ or $\mathrm{S}$ of the equator. In the ionosphere, the motion of these positively charged dust particles is relatively free along geomagnetic field lines, but is complicated by collisions with other charged particles and with neutral atoms and molecules. It will, however, be impossible for these dust particles to continue to move upwards, across the Earth's magnetic field., and so they will not reach the orbit of the International Space Station.

Next, we consider what happens remote from thunderstorms, in the fair weather region (or now called the semi-fair weather region) at latitudes greater than about 15 degrees (Rycroft et al, 2012) [14]. Here the field lines can reach from the bottom of the ionosphere up to $400 \mathrm{~km}$ altitude or more (especially for higher latitudes). The maximum latitude of the ISS is, however, about 51 degrees (the inclination of the orbit). The ionospheric electric field due to the return part of the global circuit is very weak, at the very most $\sim 10 \mu \mathrm{V} / \mathrm{m}$, and from Fig. 1 we note that the electric field at $80 \mathrm{~km}$ has this value. The force due to the electric field is thus very small and from equation (4) $\mathrm{F} \sim 10^{-23} \mathrm{~N}$. This force is vertically downward on a positive ion or upward on a negative ion. In the case of a negatively charged dust particle that was considered earlier we have from eq. (7) $\mathrm{F} / \mathrm{G} \sim 10^{-6}$, so the upward force is inadequate to overcome gravity by a very large factor. 
We conclude by remarking that the discovery of Earth-like microorganisms on the exterior of the International Space Station by Grebennikova et al [7], if confirmed, gives strong credence to their introduction to the geospace environment from outside the Earth rather than from the Earth's surface. Only an infinitesimal part of the 100 of tonnes of meteoritic and cometary dust entering the Earth on a daily basis needs to be in the form of bacteria to explain their result.

\section{References}

1. Lynch, K.A. et al (2005). Multiple scattering rocket observations of charged dust in the polar winter mesosphere., J. Geophys. Res, 110, A03302, doi:10.1029/2004JA010502.

2. Reche, I. et al (2018). Deposition rates of viruses and bacteria above the atmospheric boundary layer, The ISME Journal. (https://doi.org/10.1038/s41396-017-0042-2)

3. Khodadad, C.L. et al (2017). Stratospheric conditions inactivate bacterial endospores from a Mars spacecraft assembly facility, Astrobiology, 17(4) doi:10.1089/ast.2016.1549.

4. Smith, D.J. (2013). Microbes in the upper atmosphere and unique opportunities for astrobiology research, Astrobiology, 13, 981.

5. Imshenetsky, A.A. et al (1976). On micro-organisms of the stratosphere, Life Sci Space Res, 14, 359-362.

6. Wainwright, M. et al (2003). Microorganisms cultured from the stratospheric air samples obtained at $41 \mathrm{~km}$, FEMS Microbiol Lett, 218, 161.

7. Grebennikova, T.V., et al (2018). The DNA of bacteria of the World Ocean and the Earth in cosmic dust at the International Space Station, The Scientific World Journal. (https://www.hindawi.com/journals/tswj/aip/7360147/).

8. Narlikar, J.V. et al (2003). Detection of microorganisms at high altitude, Current Science, 88(1), 23.

9. Rycroft, M. J. et al (2008). An overview of Earth's global electric circuit and atmospheric conductivity. Space Sci. Rev. 137, 83-105 .

10. Spitzer, L., (1941). The dynamics of the interstellar medium: 1 Local equilibrium, Astrophys.J., 93,369

11. Wickramasinghe, N.C., (1967). Interstellar Grains (Chapman \& Hall, Lond.)

12. Ampferer, M. et al (2010) Decrease in the electric field penetration into the ionosphere due to low conductivity at the near ground atmospheric layer ,Ann.Geophys, 28, 779.

13. Thomas, J.N. et al (2008). Lightening-driven electric fields measured in the lower atmosphere: Implications for transient luminous events, J.Geophys.Res., 113, A12306.

14. Rycroft, M. J. et al (2012). Recent advances in global electric circuit coupling between the space environment and the troposphere, Journal of Atmospheric and Solar-Terrestrial Physics, 90-91, 198-211. 